\title{
长江口水体混合过程中溶解态稀土元素分布特征
}

\author{
王中良 刘丛强
}

(中国科学院地球化学研究所环境地球化学国家重点实验室, 贵阳 550002. Email: zlwang@gyig.ac.cn)

摘要 采用液-液萃取与反萃取分离富集方法和 ICP-MS 测试技术, 对长江河口南支盐度 $0.15 \sim$ $19 \mathrm{mg} / \mathrm{g}$ 表层水体中溶解态稀土元素(REE)的浓度进行了测定. 与世界其他河口相比, 长江口溶解 态 REE 的河水端员浓度相对偏低, 仅为 $11.963(\mathrm{Y}) \sim 0.098(\mathrm{Lu}) \mathrm{ng} / \mathrm{kg}$. 溶解态 REE 在长江口低盐 度区 $(0.15 \sim 1 \mathrm{mg} / \mathrm{g})$ 明显析出, 但其析出率相对偏低, 仅为 $47 \%(\mathrm{La}) \sim 25 \%(\mathrm{Lu})$ 左右. 由于长江口水 体较浅, 水与沉积物强烈的相互作用引起溶解态 REE 在盐度 $1 \sim 8 \mathrm{mg} / \mathrm{g}$ 区段出现浓度凸起峰. 颗 粒物的解吸或部分溶解过程导致溶解态 REE 浓度长江口中高盐度区 $(8 \sim 19 \mathrm{mg} / \mathrm{g})$ 显著递升.

关键词 长江口 稀土元素 分布 析出 解吸

作为连接河流与海洋的缓冲地带, 河口本身具有独立的物理动力学和化学动力学性质. 河流物质向开放海洋的净输出取决于它们在河口的地球化学行为. 有关河口 REE 地球化学的 研究一般都有几个基本的目的: 一是示踪决定 REE 行为的地球化学过程; 二是讨论 REE 本 身的分异行为; 三是估算河流物质输出对临近海洋 REE 通量的贡献. 野外研究和室内模拟混 合实验都显示溶解态 REE 在河口低盐度区自水体大规模析出, 析出量可达河水端员浓度的 $70 \% \sim 90 \%$ 或者近全部析出 ${ }^{[1 ~ 4]}$. 长江是世界第 3 大河, 研究长江口 REE 的行为对解释东中国 海乃至西太平洋 REE 的地球化学行为都有非常重要的意义, 对了解长江口河水与海水混合过 程以及更微观的水粒相互作用过程也很重要.

\section{1 样品采集与分析}

水样品于 1998 年 11 月 4 和 5 日采于长江河口南支, 自外高桥附近起经北槽至东经 $122^{\circ} 28$ ', 北纬 $31^{\circ} 2^{\prime}$ 附近的表层水体中 $(0.5 \sim 1 \mathrm{~m})$, 盐度 $0.15 \sim 19 \mathrm{mg} / \mathrm{g}$. 用硝酸预洗干净的聚乙烯 水袋将水样打上来，用于溶解态 REE 浓度测定的水样立即用 $0.1 \mu \mathrm{m}$ 中空纤维滤膜过滤，并用 超纯浓盐酸调 $\mathrm{pH}$ 值至 1.6 左右后存放于高致密聚乙烯塑料瓶中. 另有水样用 $0.22 \mu \mathrm{m}$ 滤膜过 滤后, 用于 $\mathrm{Si}, \mathrm{P}$, 主要阴离子和主要阳离子等的测定, 盐度、 $\mathrm{pH}$ 值、温度及溶解氧等的测定 及碱度滴定和 GPS 定位当场进行.

溶解态 REE 浓度分析主要包括以下步骤: 先用 HDEHP 和 $\mathrm{H}_{2}$ MEHP 混合萃取剂将 REE 从水溶液中萃取出来, 然后再用浓盐酸将 REE 从萃取剂中反萃出来并转化为硝酸盐用 ICP-MS 测定. 用 In 元素作内标对萃取流程进行监控, 用 $\mathrm{Cd}$ 元素作内标对测试过程中 ICP-MS 的稳 定性进行监控, 流程及试剂空白与样品同时进行. 分析方法细节见文献[5]. 整个流程在 100 级超净实验室内完成, 所用试剂皆为超纯, 实验用水为 Millipore 纯水系统生产的超纯水(18.2 $\mathrm{M} \Omega)$. 测定结果显示, 除 $\mathrm{Ce}(10 \%)$ 和 $\mathrm{La}(5 \%)$ 外, 其他稀土元素的空白都在样品最低浓度的 $2 \% \sim 3 \%$ 左右, 精度优于 $3 \%$. 分析结果列于表 1 . 
表 1 长江口溶解态 REE 浓度 $(\mathrm{ng} / \mathrm{kg})$ 及盐度值 $(\mathrm{mg} / \mathrm{g})$.

\begin{tabular}{|c|c|c|c|c|c|c|c|c|c|c|c|c|c|c|c|c|}
\hline 样品号 & 盐度 & Y & $\mathrm{La}$ & $\mathrm{Ce}$ & $\operatorname{Pr}$ & $\mathrm{Nd}$ & & $\mathrm{Eu}$ & $\mathrm{Gd}$ & $\mathrm{Tb}$ & Dy & Ho & Er & & $\mathrm{Yb}$ & $\mathrm{Lu}$ \\
\hline CJ01 & 0.15 & 3 & & & & & & & & & & & & & 632 & .098 \\
\hline & & & & & & & & & & & & & & & & \\
\hline & .38 & & & & & & & & & & & & & & & 0.074 \\
\hline CJ04 & 0.40 & 9.807 & 937 & 834 & & & & & & & & 20 & & & .494 & .078 \\
\hline CJ05 & 1.10 & 10.324 & 2.76 & 469 & 55 & & 56 & & & & & 208 & 577 & & .520 & .087 \\
\hline CJ06 & 1.60 & & & & 745 & & .797 & 214 & 7 & 164 & & 211 & & & & \\
\hline CJ07 & 0.85 & 733 & 2.174 & 0 & 0. & 2.7 & 0.609 & 62 & 0.997 & & 973 & 184 & 540 & 075 & 440 & 0.071 \\
\hline CJ08 & 3.40 & 15.672 & 4.026 & & & & & & 1 & & & 325 & & & & .134 \\
\hline СJ09 & 40 & 16. & 4.0 & & & & & & & & & & & & & 0.137 \\
\hline CJ10 & 4.90 & 14.8 & 3.64 & 6 & 0.946 & & & & & & & & & & 0.793 & 0.126 \\
\hline CJ11 & 6.00 & 12.7 & 3.3 & & & & & & & & & & & & & 0.106 \\
\hline & & & & & & & & & & & & & & & & \\
\hline & & & & & & & & & & & & & & & & 0.123 \\
\hline & .50 & & & & & & & & & & & & & & & 0173 \\
\hline & 9.20 & & & & & & & & & & & & & & & 0.137 \\
\hline & 2.00 & & & & & & & & & & & & & & & 0.209 \\
\hline CJ17 & 18.00 & 29.702 & 5.255 & 6.855 & 1.326 & 6.72 & 1.328 & 0.364 & 2.478 & & 2.504 & 0.565 & 1.654 & 0.251 & 1.579 & 0.274 \\
\hline CJ18 & 19.00 & 32.868 & 5.56 & 6.861 & 1.373 & 6.82 & 1.367 & 0.382 & 2.594 & 0.340 & 2.608 & 0.623 & 1.807 & 0.276 & 1.697 & 0.304 \\
\hline CJ19 & 16.80 & 30.147 & 5.288 & 6.859 & 1.328 & 6.527 & 1.270 & 0.369 & 2.478 & 0.334 & 2.510 & 0.573 & 1.637 & 0.246 & 1.539 & 0.267 \\
\hline
\end{tabular}

\section{2 结果与讨论}

\section{1 溶解态 REE 在长江口水体中的分布规律}

图 1 为溶解态 REE 浓度-盐度图, 清楚地表达了溶解态 REE 浓度在长江口盐度剖面上的 变化趋势(图中以 $\mathrm{Nd}$ 元素为例, 其他稀土元素的变化趋势和 $\mathrm{Nd}$ 基本一致). 在河口低盐度区, 溶解态 REE 浓度迅速降低, Nd 元素浓度由盐度 $0.15 \mathrm{mg} / \mathrm{g}$ 时的 $4.813 \mathrm{ng} / \mathrm{kg}$ 下降到盐度 $0.85 \mathrm{mg} / \mathrm{g}$ 时的 $2.703 \mathrm{ng} / \mathrm{kg}$, 说明溶解态 REE 在该区有明显的析出行为发生; 在盐度 $0.85 \sim$ $8 \mathrm{mg} / \mathrm{g}$ 区段, 溶解态 REE 浓度先升后降, 出现明显的浓度凸起峰, Nd 元素在凸起点浓度可达 $4.819 \mathrm{ng} / \mathrm{kg}$, 说明该区很可能受到强烈的水与沉积物相互作用的影响, 或者有另外的含溶解 态 REE浓度较高的水体加入; 盐度高于 $8 \mathrm{mg} / \mathrm{g}$ 以后, 溶解态 REE浓度随盐度增加线性递增, $\mathrm{Nd}$

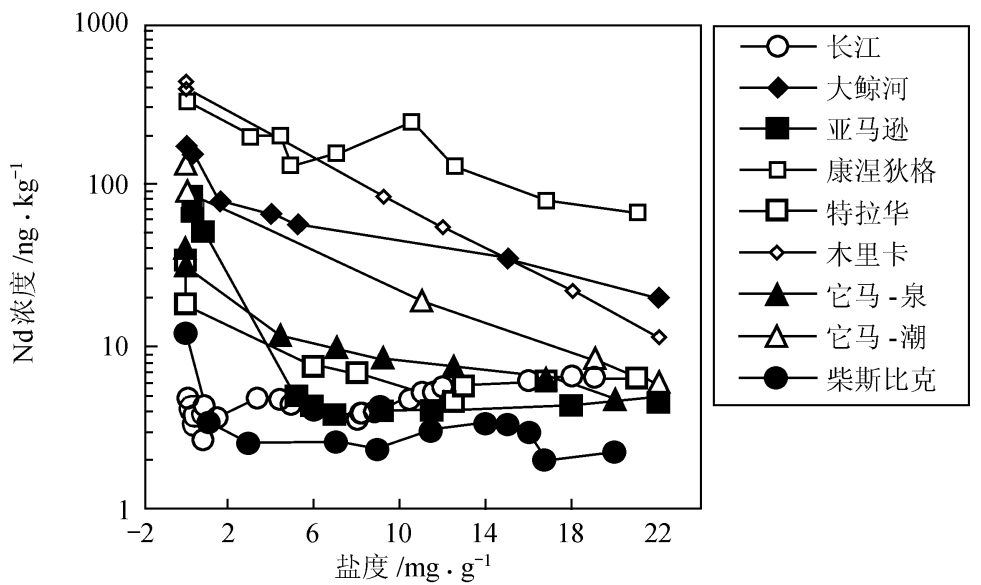

图 1 长江河口溶解态稀土元素浓度随盐度分布特征及其与世界其他河口同一盐度区的对比 长江 REE 以 $\mathrm{Nd}$ 为例, 其他河口数据引自文献 $[1 \sim 4]$ 
元素浓度由盐度 $8 \mathrm{mg} / \mathrm{g}$ 时的 $3.626 \mathrm{ng} / \mathrm{kg}$ 递升到盐度 $19 \mathrm{mg} / \mathrm{g}$ 时的 $6.824 \mathrm{ng} / \mathrm{kg}$, 说明该区有明 显的颗粒物的解吸或部分溶解现象发生. 长江口水体混合过程中, 轻稀土(LREE)和重稀土 (HREE)随盐度增加产生分异, LREE 在河口低盐度区优先析出, 而 HREE 在中高盐度区的浓 度递升较快, 分异程度随盐度增加而加深. Ce 元素相对于 $\mathrm{La}$ 和 $\mathrm{Nd}$ 也显示负异常, 而且负异 常程度也随盐度增加而增加.

与世界其他河口相比，长江河口溶解态 REE 的河水端员浓度和河口析出率都相对偏低, 河水端员浓度仅为 $11.963(\mathrm{Y}) \sim 0.098(\mathrm{Lu}) \mathrm{ng} / \mathrm{kg}$ 左右, 河口析出率也仅为 $47 \%(\mathrm{La}) \sim 25 \%(\mathrm{Lu})$ 左 右. 在以前的研究中, 溶解态 REE 在河口的浓度升高现象并不普遍, 只有巴西的亚马逊河口 在盐度高于 $6.5 \mathrm{mg} / \mathrm{g}$ 时出现溶解态 REE 浓度的规律性递升(图 1) (3] $^{[3]}$

在长江河口水体混合过程中, 北美页岩(NASC)标准化的溶解态 REE 配分模式也随盐度 发生适度变化, 特别是中稀土元素(MREE)向海水方向相对亏损, 反应了颗粒物对水体的影响 向海洋方向逐渐减弱 ${ }^{[6]}$. 整体而言, 溶解态 REE 在长江河口以 HREE 相对富集为主要特征, 并 显示了自河流向海洋的过渡特征(图 2).

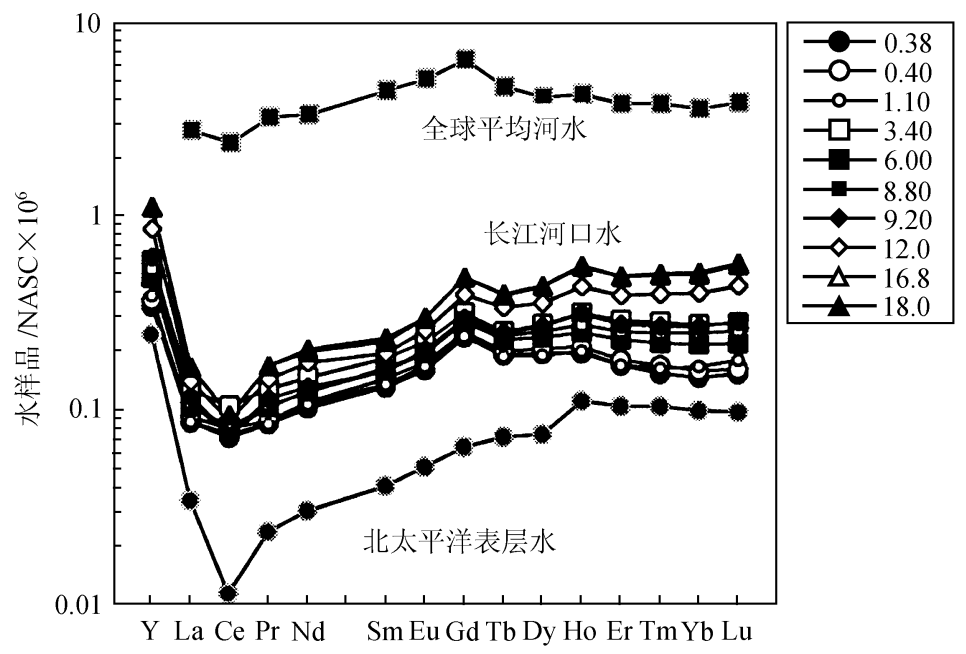

图 2 长江河口水体北美页岩 (NASC) 标准化的溶解态 REE 配分模式及其 与全球平均河水和北太平洋表水体溶解态 REE 配分模式的对比 太平洋表层水体 REE 数据引自文献[7], 右边图例所示为盐度值 $(\mathrm{mg} / \mathrm{g})$

\section{2 长江口溶解态 REE 浓度及行为的主要控制因素}

(1) 长江口溶解态 REE 河水端员浓度的控制因素. 长江河口溶解态 REE 的河水端员浓 度相对偏低, 其原因可能有以下几个方面：首先, 长江水体含有较丰富的悬浮颗粒物质, 使溶 解态 REE 的主要赋存物胶体和有机质被强烈吸附并在颗粒物迅速沉降中被大量清除, 这就降 低了溶解态 REE 浓度 ${ }^{[8]}$; 其次, 长江较高的 $\mathrm{pH}$ 值 $(7.7 \sim 8.0)$ 和较高的常量阳离子浓度 $\left(\left(\mathrm{Na}^{+}+\mathrm{Ca}^{2+}\right)\right.$ 浓度约 $\left.70 \mu \mathrm{g} / \mathrm{g}\right)$ 使胶体物质在长江水体的大量存在变得困难, 也会降低长江水体溶 解态 REE 的浓度 ${ }^{[9]}$. 同样的现象见于美国的俄亥俄河和密西西比河、巴基斯坦的印度河等, 它 们具有较高的 $\mathrm{Na}^{+}, \mathrm{Ca}^{2+}$ 离子浓度和较高的 $\mathrm{pH}$ 值, 而其溶解态 REE 浓度相对偏低 ${ }^{[10]}$. 巴西的 
亚马逊河恰恰相反, 它具有较低的 $\mathrm{Na}^{+}, \mathrm{Ca}^{2+}$ 离子浓度和较低的 $\mathrm{pH}$ 值, 但其溶解态 REE 浓度相 对偏高 ${ }^{[3,10]}$. 另外,长江充沛的水量及较短的水体驻留时间也会降低长江水溶解态 REE 的浓度. 张劲等人 ${ }^{[111}$ 对长江主航道(重庆至上海)水体溶解态 REE 浓度的测定结果也显示了较低的值.

(2) 长江口水体混合过程中的溶解态 REE行为及控制因素. 在过去近 10 年的研究中, 由 盐水加入而引起的河流胶体絮凝作为溶解态 REE 在河口低盐度区析出的根本机制已被建立 起来并得到广泛认可 ${ }^{[1 \sim 3]}$. 也有学者认为变价的 $\mathrm{Ce}$ 元素和 $\mathrm{Mn}$ 元素在河口水体混合过程中会 被氧化成惰性的 $\mathrm{Ce}(\mathrm{IV})$ 和 $\mathrm{Mn}(\mathrm{IV})$, 以 $\mathrm{CeO}_{2}$ 和 $\mathrm{MnO}_{2}$ 的形式吸附在悬浮颗粒物上析出 ${ }^{[3,12]}$. 我 们认为泥沙沉降过程也可能影响到溶解态 REE 在河口的析出行为, 特别对于长江等颗粒物含 量较高而流速变化比较大的河口, 因为我们发现实际的河口析出过程比混合实验中的析出要 迅速得多, 析出率也较大.

长江入海之初, 由于水面骤然变宽、水流变缓, 水体颗粒物迅速沉降, 同时水体主元素 浓度也清楚地显示了海水成分的加入. 所以盐水加入引起的胶体絮凝是溶解态 REE 在长江河 口低盐度区析出的主要原因, 并受到颗粒物大量沉降的影响. 由于长江较高的 $\mathrm{pH}$ 值, 使胶体 物质在长江水体的存在很不稳定, 在到达河口以前已经大量析出水体, 加之受河口区水与沉 积物强烈相互作用的影响, 所以溶解态 REE 在长江口低盐度区的析出率相对偏低.

水与沉积物的相互作用也强烈影响到长江口盐度自 $1 \sim 8 \mathrm{mg} / \mathrm{g}$ 范围内的溶解态 REE 浓度 分布, 使溶解态 REE 浓度在此区间出现明显的浓度凸起峰. 由于长江口水体较浅 (平均为 $6.5 \mathrm{~m}$ 左右)而水流混合作用相对强烈, 河口水体与底层沉积物相互作用比较强烈, 使该区悬 浮颗粒物沉降变得困难甚至使沉积物再悬浮, 从而维持了该区较高的颗粒物含量, 本研究和 Edmond 等人 ${ }^{[13]} 1981$ 年 11 月份样品的颗粒物含量都清楚地表明了这一点.

长江口盐度 $8 \mathrm{mg} / \mathrm{g}$ 以后, 溶解态 REE 浓度出现规律性递升. 这很可能是由颗粒物解吸、 部分溶解或底层沉积物释放引起, 因为大气沉降、第 3 水体端员加入或生物过程等说法都不 能对这一规律性递升现象进行很好的解释. Sholkovitz ${ }^{[3]}$ 根据亚马逊河口深水样品具有较高的 溶解态 REE 浓度认为底层释放是引起溶解态 REE 在亚马逊河口盐度 $6.5 \mathrm{mg} / \mathrm{g}$ 以后浓度递升 的主要原因, 但这种解释并不能使人完全信服, 因为吸附应该使表层水更富 HREE, 与文中所 载的深部水更富 HREE 的事实不相符, 底层来源的说法有不妥之处. 我们更倾向于溶解态 REE 在长江口中高盐度区的浓度递升是由颗粒物的解吸或部分溶解引起. 事实上, 解吸现象 在河口水体混合过程中特别是河口中高盐度区广泛存在, 如 $\mathrm{Cd}, \mathrm{Ni}, \mathrm{Zn}, \mathrm{Cu}, \mathrm{Cs}, \mathrm{Co}, \mathrm{Ba}, \mathrm{Fe}, \mathrm{Mn}$, $\mathrm{Pb}$ 和 $\mathrm{U}$ 等元素都有在中高盐度区的浓度升高现象 ${ }^{[14-17]}$. 只是由于 REE 比多数微量元素有更 强的颗粒吸附性, 它们在河口中高盐度区的解吸强度比较小, 在多数河口不易观察到. 但是作 为世界第 1 大河的亚马逊河和世界第 3 大河的长江却有足够的水量和足够的水动力条件将大 量的悬浮颗粒物带到河口中高盐度区而基本不受季节控制, 而只有在较高盐度区解吸作用才 会稳定的发生, 所以颗粒物在这两个河口中高盐度区的解吸及溶解过程比较显著 ${ }^{[17]}$. 溶解态 REE 浓度在河口中高盐度区的升高现象可能会影响到今后对河流 REE 入海通量的计算, 因为 在以前的计算中考虑较多的是河口析出过程, 对解吸及部分溶解过程考虑得较少. 事实上解 吸及部分溶解过程和胶体絮凝析出过程一样, 是决定河口溶解态 REE 行为的两个重要的非生 物过程, 只强调絮凝而不注意解吸, 可能会对微量元素在河口区的行为产生片面的理解. 


\section{3 结论}

和世界多数河口一样, 胶体絮凝引起溶解态 REE 在长江河口低盐度区析出, 这一过程在 一定程度上受到长江口强烈的泥沙沉降的影响. 受长江较高的 $\mathrm{pH}$ 值和河口区水与沉积物强 烈相互作用的影响，长江口溶解态 REE 的河水端员浓度和河口析出率相对偏低，并在一定区 段出现明显的浓度凸起峰. 受颗粒物解吸及部分溶解的影响, 溶解态 REE 浓度在长江口中高 盐度区显著递升.

致谢 感谢徐志方博士、韩贵琳女士和张云潮先生在采样过程中给予的大力协助. 本工作为国家杰出 青年科学基金(批准号: 49625304)和“攀登”计划(95-预-39)资助项目.

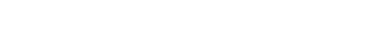

1 Elderfield H R, Upstill-Goddard R C, Sholkovitz E R. The rare earth elements in rivers, estuaries, and coastal seas and their significance to the composition of ocean waters. Geochimca Cosmochimica Acta, 1990, 54: 971 991

2 Goldstein S J, Jacobsen S B. REE in the Great Whale river estuary, northwest Quebec. Earth Plannet Sci Lett, 1988, 88: $241 \sim 252$

3 Sholkovitz E R. The geochemistry of rare earth elements in the Amazon river estuary. Geochimca Cosmochimica Acta, 1993, 57: 2181 2190

4 Sholkovitz E R, Elderfield H. Cycling of dissolved rare earth elements in Chesapeake Bay. Global Biogeochemical Cycles, 1988, 2(2): $157 \sim 176$

5 Shabani M B, Akagi T, Shimizu H, et al. Determination of trace Lanthanides and Yttrium in seawater by inductively coupled plasma mass spectrometry after preconcentration with solvent extraction and back-extraction. Anal Chem, 1990, 62: 2709 2714

张 劲, 天川裕史, 野崎义行. 太平洋西北海域水中钇与稀土元素的地球化学. 海洋与湖沼, 1996, 27(1): 51 58

7 Alibo D S, Nozaki Y. Rare earth elements in seawater: Particle association, shale-normalization, and Ce oxidation. Geochimca Cosmochimica Acta, 1999, 63:363 372

王江涛. 黄河、长江和钱塘江水体中的胶体有机碳. 科学通报, 1998, 43(8): $840 \sim 843$

9 Hu M H, Stallard R F, Edmond J M. Major ion chemistry of some large Chinese rivers. Nature, 1982, 298: 550 553

10 Goldstein S J, Jacobsen S B. Rare earth elements in river waters. Earth Planet Sci Lett, 1988, 89: 35 47

11 Zhang J, Yamada M, Wang Z L, et al. First data on REE distributions in the Changjiang water: Weathering processes and fluxes to the East China Sea. Mineralogical Magazine, 1998, A62: 1693 1694

12 Moffett J W. The relationship between cerium and manganese oxidation in the marine environment. Limnol Oceanogr, 1994, 39(6): $1309 \sim 1318$

13 Edmond J M, Spivack A, Grant B C, et al. Chemical dynamics of the Changjiang estuary. Continental Shelf Research, 4(1-2): $17 \sim 36$

14 Comans R N J, Van Dijk C P J. Role of complexation processes in cadmium mobilization during estuarine mixing. Nature, 1988, 336: 151 154

15 Li Y H, Burkhardt L, Teraoka H. Desorption and coagulation of trace elements during estuarine mixing. Geochimca Cosmochimica Acta, 1984, 48: $1879 \sim 1884$

Elbaz-Poulichet F, Holliger P, Huang W W, et al. Lead cycling in estuaries, illustrated by the Gironde estuary, France. Nature, 1984, 308: 409 414

17 Yang M, Saň udo-Wilhelmy S A. Cadmium and manganese distributions in the Hudson River estuary: Interannual and seasonal variability. Earth Planet Sci Lett, 1998, 160: 403 418 